
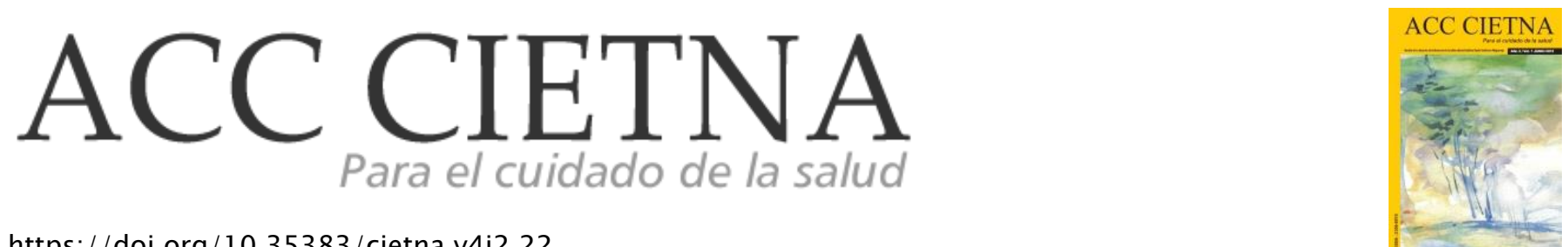

https://doi.org/10.35383/cietna.v4i2.22

\title{
Factores de riesgo del tabaquismo, consumo de tabaco en adolescentes de una Institución estatal, Chiclayo
}

\section{Coronel Fallaque Consuelo De La Paz ${ }^{1}$, Rodríguez Cruz Lisseth Dolores ${ }^{2}$}

INFORMACIÓN DEL ARTÍCULO
Historia del artículo:
Recibido el 23 de octubre de 2016
Aceptado el 15 de enero de 2017

\section{Palabras claves:}

Tabaco

Adolescencia

Factores de riesgo

\section{RESUMEN}

La adolescencia es caracterizada por cambios físicos, psicológicos, sexuales y sociales en los adolescentes, ocasionando un periodo difícil, además debido a influencias familiares o influencias sociales pueden encontrarse envueltos en problemas de salud pública como el tabaquismo. Esta investigación realizada en una institución educativa pública con el objetivo de determinar los factores de riesgo del tabaquismo y el consumo de tabaco en el adolescente. La investigación fue de tipo cuantitativa - descriptiva, la población estuvo conformada por 400 adolescentes, la muestra fue de 196 adolescentes.

La recolección de datos se realizó a través de una encuesta conformada por 28 preguntas, validada por juicio de expertos, con Alfa de Cronbach de 0,840 .

Se realizó el análisis estadístico descriptivo a través de porcentajes, frecuencias y medidas de tendencia central. El factor de riesgo de influencias familiares estuvo presente en un (24\%), las influencias de las amistades (30\%), los medios de comunicación (63\%). En cuanto al consumo de tabaco la edad de inicio fue de 14 a 15 años (8\%), el acceso a la compra de tabaco a pesar de ser menores de edad fue el $3 \%$ y en los últimos 30 días el $5 \%$ consumió tabaco. La investigación tuvo en cuenta criterios de rigor ético según Elio Sgreccia y criterios de rigor científico según Hernández. Se concluye que los adolescentes han

1 Licenciada en Enfermería del Centro de Salud María de los Sánchez Villegas - La Victoria Sector I, Chiclayo, Perú. Email: consuelocoronelfallaque@gmail.com

2 Magister en Enfermería. Docente de la Escuela de Enfermería de la Universidad Católica Santo Toribio de Mogrovejo,Chiclayo, Perú . Email: Irodriguez@usat.edu.pe 
iniciado el consumo de tabaco a edades cada vez más tempranas, en este caso es de 14 a 15 años de edad; siendo la adolescencia una etapa propensa a la curiosidad por fumar, un entorno familiar permisivo al tabaco será un incentivo para la consolidación del hábito tabáquico o el inicio de consumo.

Risk factors of tobacco, tobacco consumption in the adolescent of the state institution Abraham Valdelomar, Chiclayo, 2013

\section{ABSTRACT}

Keywords:

Tobacco

Risk factors

Adolescent.
Adolescence is characterized by physical, psychological, sexual and social changes in adolescents, causing a difficult period, also due to family influences or social influences may be involved in public health problems such as smoking. This research was carried out in the I.E. "Abraham Valdelomar; with the objective of determining the risk factors of smoking and smoking in the adolescent. The research was of quantitative - descriptive type, the population was formed by 400 adolescents, the sample was of 196 adolescents.

Data collection was done through a survey of 28 questions, validated by expert judgment, with Cronbach's alpha of 0.840 .

The descriptive statistical analysis was performed through percentages, frequencies and measures of central tendency. The risk factor for smoking, family influences was present in one (24\%), influences of friendships (30\%), media (63\%). In terms of tobacco consumption, the age of onset was 14 to 15 years (8\%), access to the purchase of tobacco despite being minors was $3 \%$ and in the last 30 days, $5 \%$ used tabaco. The research took into account criteria of ethical rigor according to Elio Sgreccia and criteria of scientific rigor according to Hernández. It is concluded that adolescents have started smoking at an earlier age, in this case it is 14 to 15 years of age; being adolescence a stage prone to the curiosity to smoke, a family environment permissive to tobacco will be an incentive for the consolidation of the smoking habit or the beginning of consumption. 


\section{Introducción}

La adolescencia es la etapa en donde la persona está descubriendo el mundo y los que están cerca del adolescente, sobre todo sus padres, vivirán todos esos cambios con cierta inquietud'. Los adolescentes pueden encontrase envueltos es el tabaquismo, reconocido en el mundo, como el causante de muertes prematuras y de enfermedades, ocasionado por malas influencias de familiares y por parte de las amistades; o por la curiosidad de fumar un cigarro. Muchos adolescentes empiezan a fumar porque lo hacen sus amigos, pero mientras más joven se inicia el consumo más probabilidades existen de hacerse adicto y sufrir las enfermedades asociadas al consumo de tabaco y morir a causa de ellas.

Los medios de comunicación ejercen también influencia en el uso de tabaco; y por causa de la falta de información los adolescentes intentan fumar por primera vez ${ }^{2}$. Los anuncios publicitarios pueden generar la confusión y el engaño en el adolescente a apoyar la idea de que el consumo de tabaco es un comportamiento razonable, sin observar las enfermedades graves y dolorosas que produce el tabaquismo.

La edad de iniciación del consumo de tabaco es a edades tempranas, aproximadamente el $75 \%$ de los fumadores iniciaron antes de alcanzar los 19 años ${ }^{3}$. Durante los últimos diez años el consumo del tabaco se ha incrementado en 31 \% en América Latina, en 41.5 \% en África y en Asia ${ }^{4}$. Los países más prevalentes al tabaquismo son aquellos países menos desarrollados iniciando el consumo de tabaco a edades tempranas, ocasionando a futuro más de 10 millones de muertes convirtiéndose en un problema de salud pública. En el Perú el inicio del consumo de tabaco se produce cada vez más en menores de edad y con gran incremento de consumo de tabaco en las mujeres. El tabaco, es la segunda drogas más consumida por la población urbana a partir de los 15 a 18 años 5 .

Otro estudio realizado en estudiantes adolescentes, refiere que uno de cada dos estudiantes ha probado alguna vez tabaco, la edad de inicio es de 13 años de edad. De acuerdo a la Ley 28705 está legalmente prohibido su venta y consumo para menores de 18 años, sin embargo las disposiciones legales son incumplidas. La venta callejera de cigarrillos de tabaco incentiva su uso sobre todo por menores de edad, sobre todo en adolescentes de sexo masculino ${ }^{6}$. Otras personas declaran que iniciaron haber consumido tabaco entre los 9 y los 18 años y el 50\% comenzó a usar este tipo de droga entre los 12 y los 15 años. El $25 \%$ empezó a consumirla antes de los 12 años?

A la luz de este análisis, la adolescencia es la etapa de mayor riesgo de inicio de consumo tabaco. Este riesgo aumenta aún si tenemos en cuenta que el riesgo de depender de estas sustancias aumenta cuando se consumen a una edad más temprana. En Lambayeque las cifras son alarmantes con un $33,24 \%$ de adolescentes consumidores ${ }^{8}$.

Muchos adolescentes empiezan a consumir tabaco a edades muy tempranas, evidenciándose que la prevalencia de tabaquismo en esta etapa de vida es muy alta. Al iniciar a edades tempranas el tabaquismo incrementará el riesgo de muerte originando también enfermedades pulmonares o cáncer. Por ello es vital que el profesional de salud conozca a profundidad las características de esta etapa de la vida, siendo un período de crecimiento y desarrollo en donde los adolescentes son más propensos a consumir sustancias tóxicas, a veces por curiosidad, y aprobación del grupo; otras por destacarse y llamar la atención de los demás, es decir, es la etapa de la vida del ser humano más susceptible para desarrollar una adicción. Está 
investigación se realizó en la Institución Estatal "Abraham Valdelomar", el director de esta institución alegó que ha sido testigo ocular que en los exteriores de la institución educativa ha aumentado el consumo de tabaco en los diferentes grados de secundaria. Además algunos estudiantes refieren que no fuman pero que tienen amigos fumadores, que a su parecer consumen tabaco por moda, para sentirse más importantes o para ser aceptados en su grupo de amigos y que adquieren cigarros sin dificultad en los vendedores ambulantes. Así mismo los padres de familia de la institución educativa manifestaron temor de que sus hijos decidan consumir tabaco y preocupación que los amigos ejerzan influencia en sus hijos.

Se considera que el trabajo de investigación es importante ya que el consumo de tabaco va en aumento en los adolescentes, el tabaquismo es considerado como epidemia mundial y a la vez también un problema social; y enfermería ocupa un lugar destacado en el abordaje del tabaquismo tanto por su accesibilidad al fumador como por su papel en la promoción de la salud y educación para la salud. La investigación tuvo como objetivo general, determinar los factores de riesgo del tabaquismo, el consumo de tabaco en el adolescente de la Institución Estatal Abraham Valdelomar -Chiclayo, 2013, y como objetivos específicos, describir los factores de riesgo del tabaquismo en el adolescente de la Institución Estatal Abraham Valdelomar -Chiclayo, 2013 y describir el consumo de tabaco en el adolescente de la Institución Estatal Abraham Valdelomar Chiclayo 2013.

\section{Metodología}

La presente investigación fue de tipo cuantitativa descriptiva9 ${ }^{9}$ porque se recopiló formación sobre los factores de riesgo del tabaquismo y el consumo de tabaco (frecuencia de consumo, el tiempo de inicio, la cantidad de consumo, los medios de acceso, lugar de consumo, persona con quien consume tabaco y gasto por consumo de cigarros) en los adolescentes de la Institución Estatal Abraham Valdelomar.

Los criterios de inclusión que se tuvieron en cuenta fueron aquellos adolescentes entre las edades de 12 a 17 años de edad, este rango de edad fue seleccionado de acuerdo a lo que señala la directiva del Ministerio de Salud de la Etapa de Vida Adolescente. $Y$ se excluyeron aquellos adolescentes cuyos padres o apoderados no consintieron la participación del estudiante en el desarrollo de la encuesta y aquellos adolescentes que no asistieron a clases en el día del desarrollo de la encuesta. El muestreo utilizado fue aleatorio simple, quiere decir; que a partir del listado de alumnos, se seleccionó al azar los participantes del estudio. El proyecto de investigación fue aprobado para su ejecución por el Comité de Ética de la Facultad de Medicina de la USAT. El instrumento de recolección de datos fue una encuesta, teniendo en cuenta el objetivo del estudio y el contexto donde se realizó la investigación utilizándose 28 preguntas de la encuesta. La encuesta fue validada por juicio de expertos, posteriormente se aplicó una prueba piloto y finalmente se midió la confiabilidad utilizando el Alfa de Cronbach de 0.840 .

Al obtener el permiso para ejecutar la investigación en la institución educativa, se tuvo acceso a las listas oficiales de matriculados durante el 2013 , se seleccionó al azar a los estudiantes, y se programó una reunión para informar respecto a la participación voluntaria y anónima en la investigación. La información recolectada fue procesada estadísticamente, los datos fueron 
vaciados en una base de datos utilizando el complemento Megastat de Excel 2010. Para el análisis de confiabilidad de los datos se calculó el coeficiente Alfa de Cronbach, basado en el promedio de las correlaciones entre los ítems. Esta medida permite la posibilidad de evaluar cuánto mejoraría (o empeoraría) la fiabilidad de la prueba si se excluyera un determinado ítem. Este trabajo de investigación tuvo en cuenta algunos de los principios éticos citados por Elio Sgreccia 10. Así tenemos: Libertad y Responsabilidad; los adolescentes fueron tratados durante toda la investigación con respeto y dignidad, tuvieron el derecho a dar por terminada su participación, de rehusarse a dar información, o de exigir que se le explique el propósito del estudio, por ello se les explicó el objetivo del estudio; se les indicó que su participación es voluntaria, la posibilidad de retirarse en cualquier momento de la investigación y su forma de participación. Se les entregó una hoja informativa, en la cual se describió detalladamente a los participantes la naturaleza del estudio, y también se les hizo la entrega de un asentimiento informado en donde ellos firmaron este documento aceptando la participación de esta investigación. Además la población de estudio fueron adolescentes siendo menores de edad, para ello se les citó a los padres o apoderados a una reunión para explicar el propósito de la investigación. Además se tuvo en cuenta la confidencialidad y anonimato esto quiere decir; que durante el trayecto de la investigación no se reveló la identidad de los adolescentes; siendo también de gran importancia de respetar la dignidad humana de los estudiantes, la igualdad, y su libertad de expresión. La calidad de un estudio está determinada en su mayoría con el rigor científico con que se realizó el trabajo de investigación, existen criterios que permiten evaluar el rigor y la calidad científica de los estudios cuantitativos. Estos criterios son: Confiabilidad, teniéndose en cuenta la coherencia metodológica, luego de medir la prueba piloto, se analizó la fiabilidad del instrumento de recolección de datos mediante el Alfa de Cronbach, obteniendo un resultado de 0,840; es decir, el instrumento es confiable y valido. El criterio de Objetividad del trabajo de investigación; dado por el esfuerzo realizado por la investigadora para que el instrumento de recolección de datos sea permeable y confiable y por último la validez de contenido; teniendo como fin en determinar la validez, se realizó el juicios de expertos, seleccionando a 3 expertos, para juzgar independientemente la bondad de los ítems del instrumento, teniendo en cuenta la congruencia del contenido, la claridad en la redacción y la tendenciosidad en la formulación de los ítems.

\section{Resultados, análisis y discusión}

El 61\% de los encuestados indicó que ninguno de sus padres fuma y solo el $7 \%$ indicó que ambos padres fuman, mientras que el $20 \%$ indica que su padre fuma, y solo el $4 \%$ de los encuestados señaló que la madre es quien fuma y el $8 \%$ indicó que desconocen si sus padres fuman ocasionalmente.

El 63\% indicó que a veces observan fumar a los actores en televisión, el $26 \%$ señaló que nunca han observado fumar a los actores en televisión, el 5\% señaló que nunca ven televisión o cine y solo el $4 \%$ indicó que ha observado fumar a los actores de manera exagerada.

Además el $68 \%$ señaló que no fumaría aunque se lo ofrezca su mejor amigo el $18 \%$ indicó que tal vez no lo haga, un $13 \%$ señaló que si se lo pidiese su mejor amigo tal vez si fumaría, y solo un $1 \%$ señaló que definitivamente si fumaría. 
El $81 \%$ de los encuestados indicó que no fuma cigarros mientras que el 8\% indicó que entre los 14 y 15 años empezaron a consumirlo, el 6\% entre los 12 y 15 años y el $5 \%$ restante a edades menores de 12 años.

Todo entorno tabáquico es un factor de riesgo reconocido, Londoño' ${ }^{1}$ señala que esta etapa es un tiempo de crecimiento y desarrollo, con adquisición de nuevas funciones en las esferas biológica, psicológica y social.

Los factores de riesgo para el consumo de tabaco constituyen un problema de salud pública, cuya iniciación generalmente ocurre en la adolescencia, situación que culmina en el hecho que en la adultez más de la mitad de sus componentes posee hábito tabáquico. La vida del adolescente se desenvuelve en medio de una serie de cambios biopsicosociales, que lo conducen a experimentar muchas situaciones de riesgo.

En este estudio se les consultó a los adolescentes sobre diversos factores de riesgo del consumo de tabaco, entre los que tenemos: familiares, sociales, demográficos y creencias. En primer lugar se analizó a los factores familiares, donde el $20 \%$ de los adolescentes indicaron que su padre o su madre fuman, además el $8 \%$ indicó que ambos padres fuman.

Londoño afirma que todo entorno tabáquico es un factor de riesgo, en el caso de los adolescentes de este estudio, el entorno tabáquico lo constituyen sus padres; convirtiéndose en un factor de riesgo para el inicio o consolidación para el hábito tabáquico.

Estos resultados son similares a los encontrados por Moreno ${ }^{12}$ quien menciona que el convivir con personas que fuman en el hogar hace más probable que los estudiantes fumen. La prevalencia de estudiantes que viven en hogares con padre o su madre fumadores $u$ otros familiares que fumen es del 24,5\%, mientras que esta proporción es sólo del $13 \%$ en los estudiantes en los que ninguno de sus progenitores es fumador.

Por otro lado otro estudio en México, refiere que el $10 \%$ de padres fuma, y el $25 \%$ que ambos padres fuman ${ }^{13}$. Aunque el presente estudio los familiares que consumen tabaco son menos del $30 \%$; no por ello deja de ser preocupante, ya que estos adolescentes tienen en su hogar un ejemplo de que el tabaco es una conducta aceptada que les puede llevar a iniciarse en el consumo de tabaco o a consolidar el hábito.

En relación al factor social para el consumo de tabaco en el adolescente; el $63 \%$ indicó que a veces ven fumar a los actores en televisión. Estas influencias sociales que realiza continuamente la publicidad de tabaco son negativas y contribuyen a que los adolescentes imiten lo que ven.

Por otro lado el $68 \%$ señaló que no fumaria si un amigo le ofrece un cigarro. Al respecto de este último punto Garzón encontró que el porcentaje de adolescentes que han probado el tabaco por ofrecimiento de un amigo es de $29,7 \%$ y en México el $22 \%$ de los adolescentes fuma por invitación de un amigo ${ }^{14}$. Por lo contrario Paniagua refiere que la publicidad de tabaco es uno de los productos más anunciados, los mensajes publicitarios más frecuentes de tabaco fueron de libertad, alegría y diversión, aventura, así como los asociados a conductas maduras y relaciones interpersonales, siendo el $21,04 \%$ de la publicidad hallada alrededor de colegios de educación secundaria ${ }^{15}$.

El factor social es un factor fundamental para iniciarse en el hábito tabáquico. La necesidad de establecer relaciones sociales puede hacer que los adolescentes se sientan obligados a consumir tabaco; para ser aceptado en un grupo social, 
donde fumar es algo normal. En otra investigación Quintero' ${ }^{16}$, menciona que la influencia de los medios de comunicación y amigos en los adolescentes tiene relación directa e indirecta con la decisión de fumar; donde el $45 \%$ son por influencia de los amigos, los $14 \%$ cantantes o actores y un $49 \%$ de influencia de los medios de comunicación.

Otro factor de riesgo para el consumo de tabaco en el adolescente que se toma en cuenta en este trabajo de investigación son las creencias que tiene el adolescente; el 64\% refiere que el consumir tabaco hace perder peso, el $83 \%$ de los encuestados indica que el consumo de tabaco es definitivamente dañino para la salud.

En México los adolescentes reconocen que el consumir tabaco ocasiona daños para la salud en un $52 \%$ y el $48 \%$ desconoce los daños que ocasiona el tabaco ${ }^{17}$. Estos resultados aunque en un menor porcentaje son similares a los encontrados en este estudio.

Por lo contrario, en Argentina el $38,0 \%$ de adolescentes respondieron a un cuestionario refiriendo que el consumir tabaco dañaba la salud, además de ser peligroso para la vida de la persona que fuma y la de otros a su alrededor y solo un adolescente $(0,1 \%)$ dijo que se trataba de algo bueno; además el $11 \%$ respondió que el fumar hacia perder de peso ya que la nicotina quitaba el hambre ${ }^{18}$. Los estudiantes adolescentes admiten que los productos del tabaco producen daños a su salud, esta situación evidencia, de manera indirecta que el adolescente consume tabaco, sabiendo que aun así es dañino para su salud. Por lo tanto, el adolescente se encuentra en un estado de apertura al conocimiento de nuevas sensaciones y experiencias, pero no tiene todavía desarrollada la capacidad para discernir entre lo bueno y lo malo de determinadas conductas, en este caso, el consumo de tabaco, y esto lo hace susceptible a que las amistades influyan al adolescente.

De acuerdo con los datos obtenidos el profesional de enfermería debe de estar informado sobre el consumo de tabaco en la adolescencia, para que realice actividades educativas para prevenir el consumo de tabaco en adolescentes, pero sobre todo estar dispuestos a promover hábitos saludables mediante actividades educativas tales como: sesiones educativas, elaboración de carteleras informativas relacionadas con el tabaquismo.

El consumo de tabaco en adolescentes es considerado un problema importante que actualmente afronta la sociedad, ya que día a día es muy común ver a adolescentes de temprana edad consumir tabaco, por tal motivo surge la necesidad de conocer la edad de inicio de consumo de tabaco, medios de acceso y venta de consumo. Así tenemos, el 8\% indicó que entre los 14 y 15 años empezaron a consumir tabaco y el $6 \%$ entre los 12 y 15 años, el 3\% indicó haber comprado cigarros en kioskos o tiendas, el $6 \%$ refiere que le vendieron cigarros a pesar de ser menor de edad.

En el Perú un trabajo de investigación realizado por CEDRO a nivel nacional, revela resultados significativos al consumo del tabaco en los jóvenes estudiantes del nivel secundario donde el $65 \%$ de los escolares de secundaria peruanos compra los cigarrillos en una tienda y el $35 \%$ restante lo adquiere fundamentalmente del comercio informal (ambulantes), $80 \%$ de los escolares fumadores habituales que compran cigarrillos en tiendas no se les negó la venta de cigarrillos a pesar de ser menores de edad 19 .

Y en Panamá el 53.6\% de los encuestados reportó que consumieron tabaco entre los 15 a 18 años, y 
el $50 \%$ de los estudiantes fumadores compraron cigarrillos en tiendas y el $\mathbf{7 6 . 9 \%}$ de los fumadores no se vieron impedidos para adquirir estos productos debido a su edad 20 .

La educación para la salud forma parte de la educación del alumnado y, por tanto, es una tarea que el profesorado debe llevar a cabo en colaboración con los profesionales de la salud. Este abordaje educativo debe centrarse, fundamentalmente, en los valores, las actitudes, las habilidades y los procedimientos, no en los contenidos conceptuales, para que el alumnado tenga criterios propios, adopte un pensamiento crítico y tome las decisiones más adecuadas para la gestión de su salud.

La mayoría de los estudiantes del colegio Abraham Valdelomar de Chiclayo, conoce las consecuencias que provoca el consumo de cigarrillo, pero no toman conciencia de ellas, y eso es lo que se tiene que lograr. Estos resultados muestran la importancia de adoptar medidas para erradicar el consumo de tabaco, por el peligro, que ello representa.Las enfermeras tenemos que iniciar la prevención a edades tempranas, acercándonos a los colegios para brindar charlas a docentes, padres de familia y a todos los estudiantes de los niveles primario y secundario y así contribuir a la prevención de los factores de riesgo del consumo del tabaco, haciéndoles ver los efectos negativos del consumo de tabaco, como son riesgo de enfermedad, discapacidad y muerte. Haciéndoles ver y tomar conciencia que los fumadores mueren a temprana edad, perdiendo en promedio de 20 a 25 años de vida productiva. Se debe conversar con los padres de familia respecto a la responsabilidad que tienen con sus hijos y que va más allá de suministrarles bienes y servicios sanitarios y educativos, a fin de orientarles para evitar el consumo de tabaco y otras drogas.
De hecho, se observa que aquellos estudiantes cuyos padres no conocen a sus amigos presentan una mayor tendencia a fumar. De hecho, la influencia de la presión social en el consumo de tabaco es más fuerte que la que ejerce otras variables. Así que para combatir el consumo de tabaco y drogas, hay que promover espacios saludables, de prácticas físicas, pues será más beneficioso para todos.

\section{Conclusiones}

Los adolescentes de este estudio cuentan con un familiar fumador (24\%). Este hecho influye en las decisiones de inicio de consumo de tabaco en los adolescentes porque al vivir en un entorno permisivo con el tabaco será un incentivo para la consolidación del hábito tabáquico o el inicio de consumo.

Los estudiantes adolescentes creen que el consumo de tabaco produce daño a su salud (79\%) y a pesar de ello tal vez consumirían tabaco si es que un amigo le ofrece un cigarro (13) por el simple hecho de establecer relaciones sociales haciendo que sus amistades influyan en él y a consecuencia de eso el adolescente iniciará el hábito tabáquico por lo cual se considera un factor de riesgo.

La creencia extendida entre los adolescentes de este estudio respecto a que el consumo de tabaco hace perder peso (68\%), no tiene sustento científico ya que el consumo de tabaco solía asociarse con la supresión del apetito, los efectos reales de la nicotina incluyen una elevación de la presión arterial y de la motilidad gástrica y enfermedades pulmonares.

En cuanto a la variable de consumo de tabaco es importante resaltar que los resultados 
encontrados en este trabajo de investigación que al igual que en otros estudios, se han encontrado que la edad de inicio para el consumo de tabaco es cada vez a edades más tempranas, en este caso es de 14 a 15 años de edad; siendo la adolescencia una etapa propensa a la curiosidad por fumar y vulnerable a situaciones para iniciar el consumo a ciertas sustancias toxicas, en este caso el tabaco.

El $3 \%$ de los adolescentes compraron cigarros fácilmente en kioskos, tiendas máquinas de cigarros o dieron dinero a alguien para que compre por ellos, aunque es un porcentaje menor este hecho nos sugiere que los adolescentes cuenta con un entorno que favorece el consumo de tabaco aunque existe una Ley que regula la venta de tabaco a los adolescentes.

\section{Bibliografía}

1. Castellano G. El adolescente y su entorno: sociedad, amigos y familia. Pediatría Integral. Santiago de Chile. 2010.

2. Jiménez J, Villar F. Factores de riesgo para el consumo de tabaco en adolescentes. Lima. Editorial Salud Pública. 2011.

3. Martínez D, Rubio M. Daños a la salud por el tabaquismo. Buenos Aires. Situación Mundial actual. 2010.

4. Organización Panamericana de la Salud. Informe sobre el control del Tabaco para las Américas .Oficina Regional de la Organización Mundial de la Salud. Wanshington DC; 2010.

5. Sánchez E. Tabaquismo en adolescentes [tesis doctoral]. Lambayeque: Primer Congreso Peruano de Educación Médica. Universidad Nacional Pedro Ruiz Gallo; 2010.

6. Castro R, Zavaleta A. Epidemiología de drogas en la población urbana peruana. Lima. Centro de Información y Educación para la Prevención del Abuso de Drogas. 2010.

7. Trujillo F.D. Informe ejecutivo "Encuesta Nacional de consumo de Drogas". Población general. Perú, 2010

8. López B.A. II Estudio Epidemiológico Andino sobre Consumo de Drogas en la Población Universitaria. Informe Perú, 2012.

9. Ñaupas P. Humberto M, Novo E, Villagómez A. Metodología de la investigación científica y asesoramiento de tesis. 2da Edición. Lima Perú.2011.

10. Sgreccia. Bioética Personalista: Principios y Orientaciones. I Congreso Internacional de Bioética. Universidad Católica Santo Toribio de Mogrovejo. Chiclayo - Perú, 14 - 16 Octubre. 2008.

11. Londoñ L. Factores relacionados al consumo de tabaco en cigarrillos en escolares adolescentes de la ciudad de Medellín, 1992.

12. Moreno R. Ana M. Tabaquismo en la adolescencia y la intervención enfermera [monografía en Internet]. Ávila: Fundación INDEX, 2014. Disponible en: http://www.indexf.com/para/n20/425.php 
13. Donas BS, Coll A. Adolescencia y Juventud en América Latina, "Marco epidemiológico conceptual de la salud integral y el desarrollo humano de los adolescentes", Edt. LUR, 2001.

14. Garzón R, Fernández E, Márquez M. Estudio de la prevalencia del tabaquismo en adolescentes de Almuñécar. Unidad de Gestión Clínica de Almuñécar, Área de Gestión Sanitaria Sur de Granada. España. 2014.

15. Paniagua $H$. Publicidad en la calle de tabaco y alcohol [sede web]*. Chile. 2004-[actualizada en el 2004; acceso 8 de julio del 2015]. Disponible: http://www.adolescenciasema.org/index.php?me $\mathrm{nu}=$ documentos $\& \mathrm{id}=42 \& \mathrm{id} \_$doc $=72 \&$ show $=1$

16. Quintero $M$. Influencia de medios de comunicación, familiares y pares sobre la decisión de fumar de universitarios. México. 2014.

17. Sánchez L, Ángeles A, Anaya R, Lazcano E. Prevalencia del uso de drogas ilegales en función del consumo de tabaco en una muestra de estudiantes en México. Salud Pública. México, 2013.

18. Luque MJ, Mesa A, Alberro J, Palma S, Cáliz A. ¿Qué piensan los adolescentes sobre el tabaco? Medicina de Familia. Argentina, Buenos Aires. 2012.

19. Centro de Información y Educación para la Prevención del Abuso de Drogas (CEDRO. Tabaquismo en escolares de secundaria: Encuesta Mundial de Tabaquismo en Jóvenes. Centros para el Control y la Prevención de Enfermedades. Organización Panamericana de la Salud - Lima: CEDRO, 2005.

20. Pamar A. Métodos educativos en salud. ELSEVIER. Barcelona, España. 2014. 